\title{
Performance Analysis of Photovoltaic Systems Using (RAMD) Analysis
}

\author{
Anas Sani Maihulla ${ }^{\mathrm{a}, *}$, Ibrahim Yusuf ${ }^{\mathrm{b}}$ \\ ${ }^{a}$ Department of Mathematics, Sokoto State University, Sokoto \\ ${ }^{b}$ Department of Mathematical Sciences, Bayero University, Kano
}

\begin{abstract}
The primary aim of this present study is to examine how reliability, availability, maintainability, and dependability (RAMD) are used to describe the criticality of each sub-assembly in grid- connected photovoltaic systems. A transition diagram of all subsystems is produced for this analysis, and Chapman-Kolmogorov differential equations for each variable of each subsystem are constructed using the Markov birth-death process. Both random failure and repair time variables have an exponential distribution and are statistically independent. A sufficient repair facility is still available with the device. The numerical results for reliability, maintainability, dependability, and steady-state availability for various photovoltaic device components have been obtained. Other metrics, such as mean time to failure (MTTF), mean time to repair (MTTR), and dependability ratio, which aid in device performance prediction, have also been measured. According to numerical analysis. it is hypothesized that subsystem $\mathrm{S} 4$, i.e. the inverter, is the most critical and highly sensitive portion that requires special attention in order to improve the efficiency of the PV device plant. The findings of this research are very useful for photovoltaic system designers and maintenance engineers.
\end{abstract}

DOI:10.46481/jnsps.2021.194

Keywords: Photovoltaic system, Mean Time to Repair, Mean Time to Failure.

Article History:

Received: 05 April 2021

Received in revised form: 26 June 2021

Accepted for publication: 30 June 2021

Published: 29 August 2021

(C)2021 Journal of the Nigerian Society of Physical Sciences. All rights reserved. Communicated by: W. A. Yahya

\section{Introduction}

The dependability of photovoltaic systems and their components/elements such as solar cells, PV modules, electrical storage systems, inverters, regulators, etc is a critical problem in production efficiency and financially competitive photovoltaic installations. A product's reliability can be described as the likelihood of system completing its tasks within a given time frame under stipulated conditions. The consistency of a product is calculated on this basis, with the reliability principle be-

\footnotetext{
${ }^{*}$ Corresponding author tel. no: $+234(0)$

Email address: anasmaihulla@gmail .com (Anas Sani Maihulla )
}

ing used in nearly all fields of engineering, including preventive maintenance of structures and their components. PV systems have become a common solution for residential houses and other autonomous applications due to numerous reward schemes and local market conditions in many European countries, as well as around the world.

RAMD is regarded as one of the most important fields for increasing profitability. RAMD modeling can help to improve safety and environmental efficiency, both of which are essential factors in every industry. Therefore, it becomes important to evaluate each and every part or subsystem of the system in order to execute safety and environmental performance. Many re- 
searchers have presented appreciable works in this regard. Researchers such as; Huffman [1], Hamdy et al. [2] and Graaff et al. [3] investigated on significant issues concerning the reliability, and safety of PV systems, RAM study of large-scale grid-connected solar-PV systems is conducted using a range of reliability approaches. Zhang et al. [4] and Hu et al. [5] conduct reliability block diagram (RBD) and fault tree analysis (FTA). In the sense of an FTA, the physical structure is translated into a logical diagram, with each block representing a device part. The failure rate is the only thing that describes each block. The overall system's reliability is calculated by the failure rates of each sub-assembly, so any failure is critical. Failure rates are frequently assumed to be constant. Gahlot et al. [6] study stochastic analysis of a two units' complex repairable system with switch and human failure using copula approach. Also recently, Chiacchio [7] investigated Dynamic performance evaluation of photovoltaic power plant by stochastic hybrid fault tree automaton model. RAMD technique was used by Saini et al. [8] and Goyal et al. [9] to define the most vulnerable aspect of serial processes such as evaporation systems in the sugar industry and water treatment plants. This study deconstructs the efficiency indices of the power generation system using STP. The power system was studied using simple probability theory principles and the Markovian birth-death process. As a Markov process proceeds from one stage to the next. Van Casteren et al. [10] used the Weibull Markovian approach to conduct consistency checks on electrical power systems. Eti et al. [11] explored a basic issue in order to maximize resource distribution in a thermal power plant. The operational availability assessment was carried out in order to improve the system's effectiveness. Ebeling [12] proposed many methods for testing the reliability and maintainability of devices with differing failure and repair rates. S. Gupta et al. [13] Studied Simulation modeling and analysis of complex system of thermal power plant. As a case study Tsarouhas et al. [14] investigated the reliability, availability, and maintainability of a strudel production line using the best suited allocation of failure and repair rates. Carazas et al. [15] proposed a framework for evaluating gas turbine power plant efficiency indicators. The study of reliability assessment of system having two subsystem in series configuration attended by human operator using Gumbel-Hougaard family copula was carried out by Singh et al. [16]. Singh [17] analyzed the performance of a system with imperfect switch consisting of two subsystem arranged in series through copula approach. Yusuf et al. [18] focus on reliability analysis of computer system configured as series-parallel system having three dissimilar subsystem via copula approach. Raghav et al. [19] dealt with reliability prediction and performance evaluation of distributed system consisting with similarity software and server architecture using joint probability distribution via Copula approach.

To address the issues raised in the previous literature on the reliability of grid-connected PV systems, this paper provides a full comprehensive RAM analysis for all sub-assemblies of grid-connected solar PV systems with a low reliability grid, taking into account failure details and repair interval (period of identification and replacement of the PV system). Furthermore, the aim of this paper is to describe the criticality of each sub-assembly of grid-connected PV systems in terms of reliability. The scope of this paper has also been broadened to establish the best probability density function for the failure rate of each solar-PV device subassembly. The rest of this paper is structured as follows; Section 2 captures materials and methods. Section 3 provides RAMD indices for PV system. Section 4 focuses on discussion of the results and the paper is concluded in Section 5.

\section{Materials and Methods}

The RAMD analysis was used in this study. RAMD is one of the most significant fields for rising profitability. RAMD modeling aids in the improvement of safety and environmental performance, both of which are critical in any industry. In this study the RAMD analysis has been performed on PV system plant. PV system mainly consists of five components namely PV modules, controller, batteries, inverter and Distribution Board. All components are arranged in series configuration. The brief description of the subsystems is given below:

1. Subsystem R (Solar module): There are two units of solar panel which is connected to the following unit in parallel. One is operational while the other one is on standby mode. Failure of the two units leads to system failure.

2. Subsystem S (Charge controller): There is one unit of charge controller which is connected to the following unit in series. Failure of this unit leads to system failure.

3. Subsystem T (Battery): This subsystem has two units of batteries which is connected in parallel to another subsystem. One is operation while the other one is in standby mode. This unit's failure causes complete system failure.

4. Subsystem U (Inverter): It consists of one unit of inverter. This unit's failure causes complete system failure as it is connected to the following unit in series.

5. Subsystem V (Distribution Board): It consists of one unit of Distribution Board. This unit's failure causes complete system failure as it is connected to the following unit in series. The RAMD analysis was focused on failure rates, and the model data in this article was established theoretically.

\subsection{Assumptions}

1. Each subsystem's failure and repair rates are distributed exponentially.

2. The Failure and repair rates are statistically independent of one another.

3. There are no concurrent faults in the subsystem.

4. There are plenty of maintenance and replacement options. Repairmen are still present in the factory, and the restored machine performs as well as new. 
Table 1. Failure and repair rates of component of the photovoltaic system

\begin{tabular}{lll}
\hline \hline Subsystem & Failure Rate $(\omega)$ & Repair Rate $(\beta)$ \\
\hline \hline$S_{1}$ & $\omega_{1}=0.001$ & $\beta_{1}=0.5$ \\
$S_{2}$ & $\omega_{2}=0.002$ & $\beta_{2}=0.7$ \\
$S_{3}$ & $\omega_{3}=0.003$ & $\beta_{3}=0.9$ \\
$S_{4}$ & $\omega_{4}=0.004$ & $\beta_{4}=1.1$ \\
$S_{5}$ & $\omega_{5}=0.005$ & $\beta_{5}=1.3$ \\
\hline
\end{tabular}

\subsection{Notation}

$R, S, T, U$, and $V$ Represent states under which the subsystem is operating at maximum ability. $r, s, t, u$, and $v$ reflect the conditions in which a subsystem has broken.

System is working with full capacity

System is in failure state

\section{RAMD indices for PV system}

\subsection{RAMD indices for PV modules}

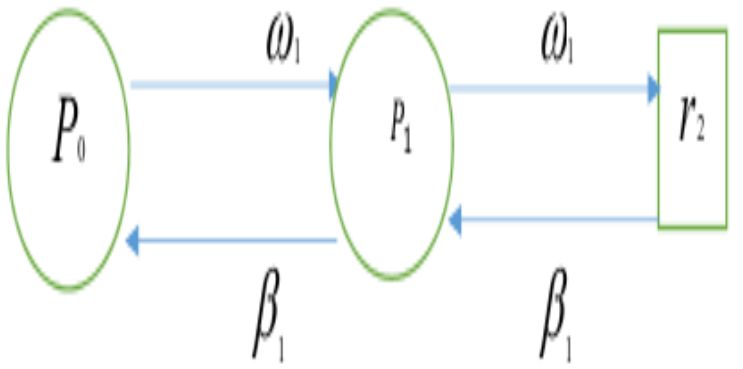

Figure 1. Transition diagram PV modules

$$
\begin{aligned}
& \frac{d}{d t} P_{0}(t)=-\omega_{1} P_{0}+\beta_{1} P_{1} \\
& \frac{d}{d t} P_{1}(t)=-\left(\beta_{1}+\omega_{1}\right) P_{1}+\omega_{1} P_{0}+\beta_{1} P_{2}
\end{aligned}
$$

$$
\frac{d}{d t} P_{2}(t)=-\beta_{1} P_{2}+\omega_{1} P_{1}
$$

Under steady state, equations (1) - (3) yield

$$
P_{1}=\frac{\beta_{1}}{\omega_{1}} P_{2}
$$

Substituting equation (4) into (1) under steady state we have:

$$
P_{2}=\frac{\omega_{1}^{2}}{\beta_{1}^{2}} P_{0}
$$

Substituting (5) into (3) under steady state we have

$$
P_{1}=\frac{\omega_{1}}{\beta_{1}} P_{0}
$$

Using normalization condition

$$
P_{0}+P_{1}+P_{2}=1
$$

It follows that:

$$
P_{0}=\frac{\beta_{1}^{2}}{\beta_{1}^{2}+\omega_{1}^{2}+\beta_{1} \omega_{1}}
$$

\subsection{RAMD indices for subsystem $R$}

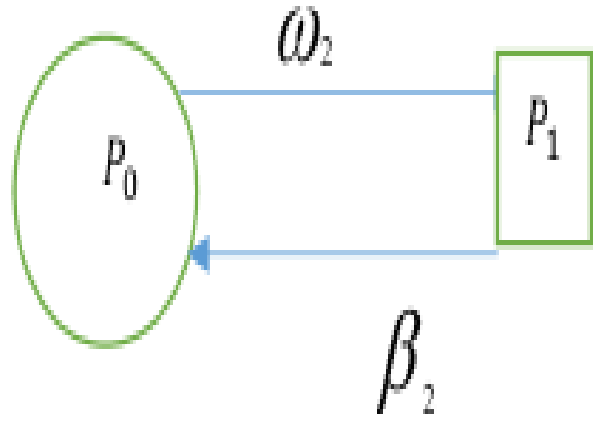

Figure 2. Transition diagram for subsystem $\mathrm{R}$

$$
\begin{aligned}
& \frac{d}{d t} P_{0}(t)=-\omega_{2} P_{0}+\beta_{2} P_{1} \\
& \frac{d}{d t} P_{1}(t)=-\beta_{2} P_{1}+\omega_{2} P_{0}
\end{aligned}
$$

Under steady state equations (8) and (9) reduces to:

$$
P_{1}=\frac{\omega_{2}}{\beta_{2}} P_{0}
$$

Using normalization condition

$$
P_{0}+P_{1}=1
$$

It follows that

$$
P_{0}=\frac{\beta_{2}}{\beta_{2}+\omega_{2}}
$$


Table 2. Variation of Reliability of subsystems with time

\begin{tabular}{ccccccc}
\hline Time (in days) & $R_{s 1}(t)$ & $R_{s 2}(t)$ & $R_{s 3}(t)$ & $R_{s 4}(t)$ & $R_{s 5}(t)$ & $R_{s y s}(t)$ \\
\hline 0 & 1.00000 & 1.00000 & 1.00000 & 1.00000 & 1.00000 & 1.00000 \\
30 & 0.92774 & 0.91393 & 0.90032 & 0.88692 & 0.87372 & 0.59155 \\
60 & 0.86071 & 0.83527 & 0.81058 & 0.78663 & 0.76338 & 0.34994 \\
90 & 0.79852 & 0.76338 & 0.72979 & 0.69768 & 0.66698 & 0.20701 \\
120 & 0.74082 & 0.69768 & 0.65705 & 0.61878 & 0.58275 & 0.12246 \\
150 & 0.68729 & 0.63763 & 0.59156 & 0.54881 & 0.50916 & 0.07244 \\
180 & 0.63763 & 0.58275 & 0.53259 & 0.48675 & 0.44486 & 0.04285 \\
210 & 0.59156 & 0.53259 & 0.47951 & 0.43171 & 0.38868 & 0.02535 \\
240 & 0.54881 & 0.48675 & 0.43171 & 0.38290 & 0.33960 & 0.01500 \\
270 & 0.50916 & 0.44486 & 0.38868 & 0.33960 & 0.29671 & 0.00887 \\
300 & 0.47237 & 0.40657 & 0.34994 & 0.30120 & 0.25924 & 0.00525 \\
330 & 0.43823 & 0.37158 & 0.31506 & 0.26714 & 0.22650 & 0.00310 \\
360 & 0.40657 & 0.33960 & 0.28365 & 0.23693 & 0.19790 & 0.00184 \\
\hline
\end{tabular}

Table 3. Variation of maintainability of subsystem with time.

\begin{tabular}{ccccccc}
\hline Time (in days) & $M_{s 1}(t)$ & $M_{s 2}(t)$ & $M_{s 3}(t)$ & $M_{s 4}(t)$ & $M_{s 5}(t)$ & $M_{S y s}(t)$ \\
\hline 0 & 0.00000 & 0.00000 & 0.00000 & 0.00000 & 0.00000 & 0.00000 \\
30 & 0.77687 & 0.95021 & 0.98889 & 0.99752 & 0.99945 & 0.72778 \\
60 & 0.95021 & 0.99752 & 0.99988 & 0.99999 & 1.00000 & 0.94773 \\
90 & 0.98890 & 0.99988 & 1.00000 & 1.00000 & 1.00000 & 0.98876 \\
120 & 0.99752 & 0.99998 & 1.00000 & 1.00000 & 1.00000 & 0.99750 \\
150 & 0.99945 & 0.99999 & 1.00000 & 1.00000 & 1.00000 & 0.99944 \\
180 & 0.99988 & 1.00000 & 1.00000 & 1.00000 & 1.00000 & 0.99988 \\
210 & 0.99997 & 1.00000 & 1.00000 & 1.00000 & 1.00000 & 0.99997 \\
240 & 0.99998 & 1.00000 & 1.00000 & 1.00000 & 1.00000 & 0.99998 \\
270 & 1.00000 & 1.00000 & 1.00000 & 1.00000 & 1.00000 & 1.00000 \\
300 & 1.00000 & 1.00000 & 1.00000 & 1.00000 & 1.00000 & 1.00000 \\
330 & 1.00000 & 1.00000 & 1.00000 & 1.00000 & 1.00000 & 1.00000 \\
360 & 1.00000 & 1.00000 & 1.00000 & 1.00000 & 1.00000 & 1.00000 \\
\hline \hline
\end{tabular}

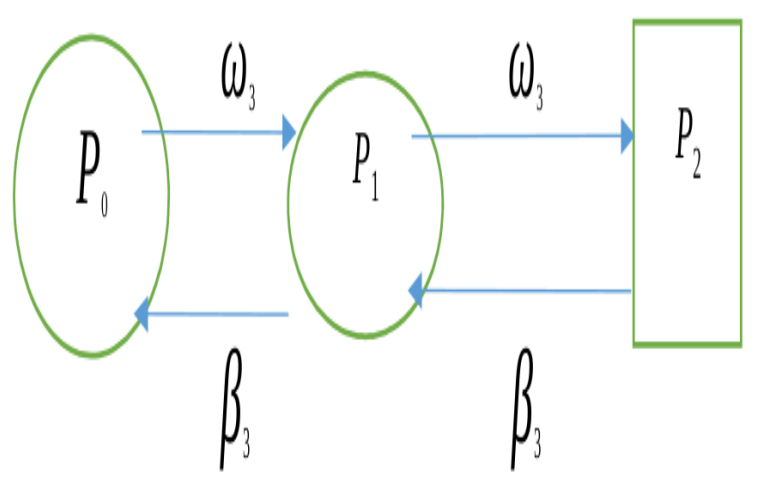

Figure 3. Transition diagram for subsystem $\mathrm{R}$

3.3. RAMD indices for subsystem $T$

$$
\begin{aligned}
& \frac{d}{d t} P_{0}(t)=-\omega_{3} P_{0}+\beta_{3} P_{1} \\
& \frac{d}{d t} P_{1}(t)=-\left(\beta_{3}+\omega_{3}\right) P_{1}+\omega_{3} P_{0}+\beta_{3} P_{2}
\end{aligned}
$$

$$
\frac{d}{d t} P_{2}(t)=-\beta_{3} P_{2}+\omega_{3} P_{1}
$$

Under steady state, equation (1) - (3) yield

$$
P_{1}=\frac{\beta_{3}}{\omega_{3}} P_{2}
$$

Substituting 4 into 1 under steady state we have:

$$
P_{2}=\frac{\omega_{3}^{2}}{\beta_{3}^{2}} P_{0}
$$

Substituting 5 into 3 under steady state we have

$$
P_{1}=\frac{\omega_{3}}{\beta_{3}} P_{0}
$$

Using normalization condition

$$
P_{0}+P_{1}+P_{2}=1
$$

It follows that:

$$
P_{0}=\frac{\beta_{3}^{2}}{\beta_{3}^{2}+\omega_{3}^{2}+\beta_{3} \omega_{3}}
$$


Table 4. RAMD indices for the photovoltaic system

\begin{tabular}{|c|c|c|c|c|c|c|}
\hline RAMD indices ofSubsystems & PV modules $S_{1}$ & Charge $\mathrm{crS} S_{2}$ & Battery bank $S_{3}$ & ${\text { Inverter } S_{4}}_{4}$ & Distributionboard & PV System \\
\hline Reliability & $e^{-0.0025 t}$ & $e^{-0.003 t}$ & $e^{-0.003 t}$ & $e^{-0.004 t}$ & $e^{-0.0045 t}$ & $e^{-0.0175 t}$ \\
\hline Maintainability & $1-e^{-0.05 t}$ & $1-e^{-0.1 t}$ & $1-e^{-0.15 t}$ & $1-e^{-0.2 t}$ & $1-e^{-0.25 t}$ & $1-e^{-0.75 t}$ \\
\hline Availability & 0.76923 & 0.97087 & 0.97668 & 0.98039 & 0.98232 & 0.70246 \\
\hline MTBF & 400 & 333.33 & 285.71 & 250 & 222.22 & 1491.27 \\
\hline MTTR & 20 & 10 & 6.67 & 5 & 4 & 45.67 \\
\hline Dependability & 0.95729 & 0.97308 & 0.97867 & 0.98153 & 0.98328 & 0.87985 \\
\hline Dependability ratio & 20 & 33.33 & 42.86 & 50 & 55.56 & \\
\hline
\end{tabular}

Table 5. Variation in system reliability as a result of PV Module failure rate variation

\begin{tabular}{ccccc}
\hline & PV System & PV System & PV modules & PV modules \\
\hline Time (in days) & $\omega_{1}=0.0011$ & $\omega_{1}=0.0012$ & $\omega_{1}=0.0011$ & $\omega_{1}=0.0012$ \\
0 & 1.00000 & 1.00000 & 1.00000 & 1.00000 \\
30 & 0.61693 & 0.62438 & 0.96754 & 0.96464 \\
60 & 0.38060 & 0.38985 & 0.93613 & 0.93053 \\
90 & 0.23480 & 0.24341 & 0.90574 & 0.89763 \\
120 & 0.14486 & 0.15198 & 0.87634 & 0.86589 \\
150 & 0.08937 & 0.09489 & 0.84790 & 0.83527 \\
180 & 0.05513 & 0.05925 & 0.82037 & 0.80574 \\
210 & 0.03401 & 0.03700 & 0.79374 & 0.77724 \\
240 & 0.02098 & 0.02310 & 0.76797 & 0.74976 \\
270 & 0.01295 & 0.01442 & 0.74304 & 0.72325 \\
300 & 0.00799 & 0.00901 & 0.71892 & 0.69768 \\
330 & 0.00493 & 0.00562 & 0.69559 & 0.67301 \\
360 & 0.00304 & 0.00351 & 0.67301 & 0.64921 \\
\hline
\end{tabular}

3.4. RAMD indices for subsystem $U$

$$
\begin{aligned}
& \frac{d}{d t} P_{0}(t)=-\omega_{4} P_{0}+\beta_{4} P_{1} \\
& \frac{d}{d t} P_{1}(t)=-\beta_{4} P_{1}+\omega_{4} P_{0}
\end{aligned}
$$

Under steady state equations (8) and (9) reduces to:

$$
P_{1}=\frac{\omega_{4}}{\beta_{4}} P_{0}
$$

Using normalization condition

$$
P_{0}+P_{1}=1
$$

It follows that

$$
P_{0}=\frac{\beta_{4}}{\beta_{4}+\omega_{4}}
$$

3.5. RAMD indices for subsystem $V$

$$
\frac{d}{d t} P_{0}(t)=-\omega_{5} P_{0}+\beta_{5} P_{1}
$$

$$
\frac{d}{d t} P_{1}(t)=-\beta_{5} P_{1}+\omega_{5} P_{0}
$$

Under steady state equations (8) and (9) reduces to:

$$
P_{1}=\frac{\omega_{5}}{\beta_{5}} P_{0}
$$

Using normalization condition

$$
P_{0}+P_{1}=1
$$

It follows that

$$
P_{0}=\frac{\beta_{5}}{\beta_{5}+\omega_{5}}
$$

Using the following equations

$$
R(t)=\int_{t}^{\infty} f(x) d x \text { Reliability function }
$$

$$
\begin{aligned}
\text { Availability } & =\frac{\text { Life time }}{\text { Total time }}=\frac{\text { Life time }}{\text { Life time }+ \text { Repair time }} \\
& =\frac{M T T F}{M T T F+M T T R}
\end{aligned}
$$


Table 6. Variation in system reliability as a result of charge controller failure rate variation

\begin{tabular}{ccccc}
\hline & PV System & PV System & charge controller & charge controller \\
\hline Time (in days) & $\omega_{2}=0.0013$ & $\omega_{2}=0.0014$ & $\omega_{2}=0.0013$ & $\omega_{2}=0.0014$ \\
0 & 1.00000 & 1.00000 & 1.00000 & 1.00000 \\
30 & 0.62251 & 0.62064 & 0.96175 & 0.95887 \\
60 & 0.38752 & 0.38520 & 0.92496 & 0.91943 \\
90 & 0.24123 & 0.23907 & 0.88959 & 0.88161 \\
120 & 0.15017 & 0.14838 & 0.85556 & 0.84535 \\
150 & 0.09348 & 0.09209 & 0.82283 & 0.81058 \\
180 & 0.05820 & 0.05715 & 0.79136 & 0.77724 \\
210 & 0.03623 & 0.03547 & 0.76109 & 0.74528 \\
240 & 0.02255 & 0.02202 & 0.73198 & 0.71462 \\
270 & 0.01404 & 0.01367 & 0.70398 & 0.68523 \\
300 & 0.00877 & 0.00848 & 0.67706 & 0.65705 \\
330 & 0.00544 & 0.00526 & 0.65116 & 0.63002 \\
360 & 0.00339 & 0.00327 & 0.62625 & 0.60411 \\
\hline
\end{tabular}

Table 7. Variation in reliability of system due to variation in failure rate of Battery bank

\begin{tabular}{ccccc}
\hline & PV System & PV System & Battery bank & Battery bank \\
\hline Time (in days) & $\omega_{3}=0.0015$ & $\omega_{3}=0.0016$ & $\omega_{3}=0.0015$ & $\omega_{3}=0.0016$ \\
0 & 1.00000 & 1.00000 & 1.00000 & 1.00000 \\
30 & 0.62414 & 0.62625 & 0.95600 & 0.95313 \\
60 & 0.36455 & 0.39219 & 0.91393 & 0.90846 \\
90 & 0.24783 & 0.24561 & 0.87372 & 0.86589 \\
120 & 0.15567 & 0.15382 & 0.83527 & 0.82531 \\
150 & 0.09778 & 0.09633 & 0.79852 & 0.78663 \\
180 & 0.06142 & 0.06033 & 0.76338 & 0.74976 \\
210 & 0.03858 & 0.03778 & 0.72979 & 0.71462 \\
240 & 0.02423 & 0.02366 & 0.69768 & 0.68113 \\
270 & 0.01522 & 0.01482 & 0.66698 & 0.64921 \\
300 & 0.00956 & 0.00928 & 0.63763 & 0.61878 \\
330 & 0.00606 & 0.00581 & 0.60957 & 0.58978 \\
360 & 0.00377 & 0.00364 & 0.58275 & 0.56214
\end{tabular}

Table 8. Variation in reliability of system due to variation in failure rate of inverter

\begin{tabular}{ccccc}
\hline & PV System & PV System & Inverter & Inverter \\
\hline Time (in days) & $\omega_{4}=0.0017$ & $\omega_{4}=0.0018$ & $\omega_{4}=0.0017$ & $\omega_{4}=0.0018$ \\
0 & 1.00000 & 1.00000 & 1.00000 & 1.00000 \\
30 & 0.60230 & 0.63192 & 0.95028 & 0.94743 \\
60 & 0.36277 & 0.39932 & 0.90303 & 0.89763 \\
90 & 0.21850 & 0.25233 & 0.85813 & 0.85044 \\
120 & 0.13160 & 0.15945 & 0.81546 & 0.80574 \\
150 & 0.07926 & 0.10076 & 0.77492 & 0.76338 \\
180 & 0.04774 & 0.06367 & 0.73639 & 0.72325 \\
210 & 0.02875 & 0.04024 & 0.69977 & 0.68523 \\
240 & 0.01732 & 0.02543 & 0.66498 & 0.64921 \\
270 & 0.01043 & 0.01607 & 0.63192 & 0.61508 \\
300 & 0.00628 & 0.01562 & 0.60050 & 0.58275 \\
330 & 0.00378 & 0.00642 & 0.57064 & 0.55211 \\
360 & 0.00228 & 0.00405 & 0.54227 & 0.52310 \\
\hline
\end{tabular}

$M(t)=1-e^{\left(-\frac{-t}{M T T R}\right)} \quad$ Maintainability function

$M T B F=\int_{0}^{\infty} R(t) d t=\int_{0}^{\infty} e^{-\theta t}=\frac{1}{\theta}$
(29)

(30)
MTBF $=$ Mean Time between Failure

$$
\operatorname{MTTR}=\frac{1}{\beta} \quad \text { Mean Time to Repair }
$$


Table 9. Variation in system reliability due to variation in delivery board failure rate

\begin{tabular}{ccccc}
\hline & PV System & PV System & distribution board & distribution board \\
\hline Time (in days) & $\omega_{5}=0.0019$ & $\omega_{5}=0.002$ & $\omega_{5}=0.0019$ & $\omega_{5}=0.002$ \\
0 & 1.00000 & 1.00000 & 1.00000 & 1.00000 \\
30 & 0.63954 & 0.63763 & 0.94460 & 0.94176 \\
60 & 0.40902 & 0.40657 & 0.89226 & 0.88692 \\
90 & 0.26158 & 0.25924 & 0.84282 & 0.83527 \\
120 & 0.16729 & 0.16530 & 0.79612 & 0.78663 \\
150 & 0.19700 & 0.10540 & 0.75201 & 0.74082 \\
180 & 0.06843 & 0.06721 & 0.71035 & 0.69768 \\
210 & 0.04376 & 0.04285 & 0.67100 & 0.65705 \\
240 & 0.02799 & 0.02732 & 0.63381 & 0.61878 \\
270 & 0.01790 & 0.01742 & 0.59870 & 0.58275 \\
300 & 0.01145 & 0.01111 & 0.56553 & 0.54881 \\
330 & 0.00732 & 0.00708 & 0.53420 & 0.51685 \\
360 & 0.00468 & 0.00452 & 0.50459 & 0.48675 \\
\hline
\end{tabular}

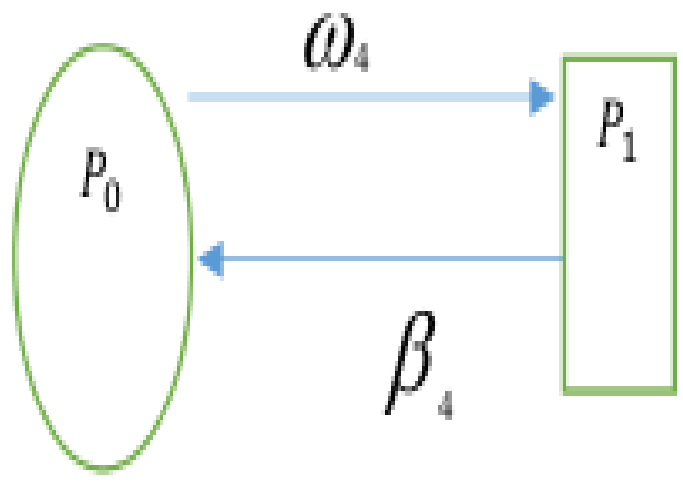

Figure 4. Transition diagram for subsystem $\mathrm{U}$

$\beta=$ Repair rate,$\omega=$ Failure rate

$$
\begin{aligned}
& d=\frac{\xi}{\omega}=\frac{M T B F}{M T T R} \\
& D_{\text {min }}=1-\left(\frac{1}{d-1}\right)\left(e^{-\frac{\ln d}{d-1}}-e^{-\frac{d \ln d}{d-1}}\right)
\end{aligned}
$$

\section{RAMD Analysis}

\section{System Reliability}

$$
\begin{aligned}
& R_{\text {sys }}(t)=R_{s 1}(t) \times R_{s 2}(t) \times R_{s 3}(t) \times R_{s 4}(t) \times R_{s 5}(t) \\
& =e^{-\left(\omega_{1}+\omega_{2}+\omega_{3}+\omega_{4}\right) t}
\end{aligned}
$$

System Availability

$$
A_{\text {sys }}=A_{s 1} \times A_{s 2} \times A_{s 3} \times A_{s 4} \times A_{s 5}
$$

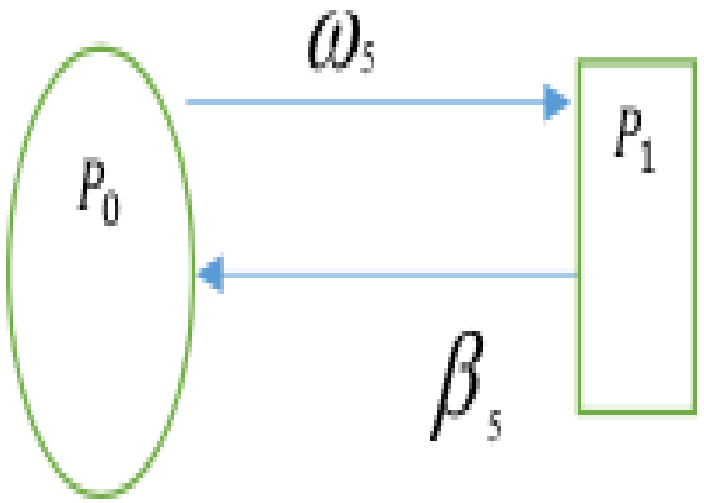

Figure 5. Transition diagram for subsystem V

System Maintainability

$$
\begin{aligned}
M_{s y s}(t) & =M_{S 1}(t) \cdot M_{s 2}(t) \cdot M_{s 3}(t) \cdot M_{s 4}(t) \cdot M_{s 5}(t) \\
& =\left(1-e^{-\beta_{1}(t)}\right) \times\left(1-e^{-\beta_{2}(t)}\right) \times\left(1-e^{-\beta_{3}(t)}\right) \times\left(1-e^{-\beta_{4}(t)}\right) \\
& \times\left(1-e^{-\beta_{5}(t)}\right) \\
& =1-e^{-\left(\beta_{1}+\beta_{2}+\beta_{3}+\beta_{4}+\beta_{5}\right) t}
\end{aligned}
$$

System dependability

$$
D_{\min (s y s)}=D_{\min (s 1)} \times D_{\min (s 2)} \times D_{\min (s 3)} \times D_{\min (s 4)} \times D_{\min (s 5)}(35)
$$

System dependability

$$
\begin{aligned}
& D_{\min (s y s)}=D_{\min (s 1)} \times D_{\min (s 2)} \times D_{\min (s 3)} \times D_{\min (s 4)} \\
& D_{\min }=1-\left(\frac{1}{d-1}\right)\left(e^{-\frac{\ln d}{d-1}}-e^{-\frac{d \ln d}{d-1}}\right) \\
& d=\frac{\beta}{\omega}=\frac{M T B F}{M T T R} \\
& d_{1}=\frac{\beta_{1}}{\omega_{1}}=\frac{0.05}{0.0025}=20
\end{aligned}
$$




$$
\begin{aligned}
& d_{2}=\frac{\beta_{2}}{\omega_{2}}=\frac{0.1}{0.003}=33.33 \\
& d_{3}=\frac{\beta_{3}}{\omega_{3}}=\frac{0.15}{0.0035}=42.86 \\
& d_{4}=\frac{\beta_{4}}{\omega_{4}}=\frac{0.2}{0.004}=50 \\
& d_{5}=\frac{\beta_{5}}{\omega_{5}}=\frac{0.2}{0.004}=55.56 \\
& D_{\min (s 1)}=1-\left(\frac{1}{20-1}\right)\left(e^{-\frac{\ln 20}{20-1}}-e^{-\frac{20 \ln 20}{20-1}}\right) \\
& D_{\min (s 1)}=0.95729 \\
& D_{\min (s 2)}=1-\left(\frac{1}{33.33-1}\right)\left(e^{-\frac{\ln 33.33}{33.33-1}}-e^{-\frac{33.33 \ln 33.33}{33.33-1}}\right) \\
& D_{\min (s 2)}=0.97308 \\
& D_{\min (s 3)}=1-\left(\frac{1}{42.86-1}\right)\left(e^{-\frac{\ln 42.86}{42.86-1}}-e^{-\frac{42.86 \ln 42.86}{42.86-1}}\right) \\
& D_{\min (s 3)}=0.97867 \\
& D_{\min (s 4)}=1-\left(\frac{1}{50-1}\right)\left(e^{-\frac{\ln 50}{50-1}}-e^{-\frac{50 \ln 50}{50-1}}\right) \\
& D_{\min (s 4)}=0.98153 \\
& D_{\min (s 5)}=1-\left(\frac{1}{55.56-1}\right)\left(e^{-\frac{\ln 55.56}{55.56-1}}-e^{-\frac{55.56 \ln 55.56}{55.56-1}}\right) \\
& D_{\min (s 5)}=0.98328 \\
& D_{\min (\text { sys })}=0.95729 \times 0.97308 \times 0.97867 \\
& \times 0.98153 \times 0.98328 \\
& D_{\min (\text { sys })}=0.87985
\end{aligned}
$$

System availability

$$
\begin{aligned}
& A_{\text {sys }}=\left(\frac{\beta_{1}^{2}}{\beta_{1}^{2}+\omega_{1}^{2}+\omega_{1} \beta_{1}}\right) \times\left(\frac{\beta_{2}}{\beta_{2}+\omega_{2}}\right) \\
& \times\left(\frac{\beta_{3}^{2}}{\beta_{3}^{2}+\omega_{3}^{2}+\omega_{3} \beta_{3}}\right) \\
& \times\left(\frac{\beta_{4}}{\beta_{4}+\omega_{4}}\right) \times\left(\frac{\beta_{5}}{\beta_{5}+\omega_{5}}\right) \\
& A_{\text {sys }}=\left(\frac{(0.05)^{2}}{(0.05)^{2}+(0.025)^{2}+(0.05) \times(0.0025)}\right) \\
& \times\left(\frac{0.1}{0.10+0.0030}\right) \times \\
& \left(\frac{(0.05)^{2}}{(0.05)^{2}+(0.0035)^{2}+(0.15)(0.0035)}\right) \\
& \times\left(\frac{0.20}{0.20+0.0040}\right)\left(\frac{0.25}{0.25+0.0045}\right) \\
& A_{s y s}=0.76923 \times 0.97087 \times 0.97668 \times 0.98039 \times 0.98232 \\
& A_{\text {sys }}=0.70246
\end{aligned}
$$

\section{Discussion}

To reflect the effect of system factors, we established formulations for availability, reliability, maintainability, and dependability for each subsystem of the model under consideration. Table 2 shows that after 300 days of operation, the PV device plant's reliability stays 0.00525 , however the distribution board's reliability is significantly poor (0.25924) across all subsystems. This sensitivity analysis reveals that the distribution board's reliability of the system requires special attention and close monitoring. As a result, machine designers must develop a maintenance plan. Tables 5- 9 showed how the reliability behavior of different subsystems improved over time and with varying failure rates.

\section{Conclusion}

In this paper, an analytical analysis for a case was performed to obtain the reliability metrics of the various subsystems and procedure as presented in Table 1. Tables 2 and 3 demonstrate the findings for the reliability and maintenance habits of various subsystems, respectively. Table 4 summarizes the remaining RAMD phases. Based on this study, it is hypothesized that subsystem S4, i.e. the inverter, is the most critical and highly sensitive portion that requires special attention in order to improve the efficiency of the PV device plant. Hence it is concluded that machine designers must develop a maintenance plan for the inverter to avoid total breakdown of the whole system and some techniques can be opted to enhance the system reliability. This research will show the method's utility and could also be used by process engineers to learn how to apply RAMD concepts to process design. Real data acquisition in working environments will be the focus of future study.

\section{References}

[1] D. L. Huffman \& F. Antelme, "Availability analysis of a solar power system with graceful degradation", In: Proceedings of the Reliability and Maintainability Symposium, Fort Worth, TX (2009).

[2] M.A. Hamdy, M. E. Beshir \& S. E. Elmasry, "Reliability analysis of photovoltaic systems", Appl. Energy 33 (1989) 253.

[3] D. De Graaff, R. Lacerda \& Z. Campeau, "Degradation mechanisms in Si module technologies observed in the field; their analysis and statistics", In: Presentation at PV Module Reliability Workshop; NREL. Denver, Golden, USA (2011).

[4] P. Zhang, W. Li, S. Li, Y. Wang \& W. Xiao, "Reliability assessment of photovoltaic power systems: Review of current status and future perspectives" Appl. Energy 104 (2013) 822.

[5] R. Hu, J. Mi, T. Hu, M. Fu \& P. Yang, "Reliability research for PV system using BDD- based fault tree analysis. In Proceedings of the 2013 International Conference on Quality, Reliability, Risk, Maintenance, and Safety Engineering (QR2MSE)", Chengdu, China (2013) 359.

[6] M. Gahlot, H.I. Agaya, V.V. Singh \& I. Abdullahi, "Stochastic analysis of a two units' complex repairable system with switch and human failure using copula approach", Life circle Reliability and safety Engineering 9 (2020) 1.

[7] F. Chiacchio, F. Famoso, D. D’Urso, S. Brusca, J.I. Aizpurua \& L. Cedola, "Dynamic performance evaluation of photovoltaic power plant by stochastic hybrid fault tree automaton model", Energies 11 (2018) 1 https://doi.org/10.3390/en11020306

[8] Saini, M. \& A. Kumar, "Performance analysis of evaporation system in sugar industry using RAMD analysis", J Braz. Soc. Mech. Sci. Eng. 41 (2019) 175. https://doi.org/10.1007/s40430-019-1681-3 
[9] D. Goyal, A. Kumar, M. Saini \& H. Joshi, "Reliability, maintainability and sensitivity analysis of physical processing unit of sewage treatment plant" SN Appl. Sci. 1 (2019) 1507 https://doi.org/10.1007/s42452-0191544-7

[10] J. F. L. Van Casteren, M. H. J. Bollen \& M. E. Schmieg, "Reliability assessment in electrical power systems: the weibull-markov stochastic model", IEEE Transactions on Industry Appl. 36 (2000) 911

[11] M. C. Eti, S. O. T. Ogaji \& S. D. Probert, "Reliability of the Afam electric power generating station, Nigeria", Applied Energy 77 (2004) 309.

[12] C. Ebeling, An introduction to reliability and maintainability engineering, 10th edn. Tata McGraw-Hill, New Delhi, Published by McGraw-Hill (2008).

[13] S. Gupta \& P.C. Tewari, "Simulation modeling and analysis of complex system of thermal power plant", J. of Industrial Eng. and Management 2 (2009) 387.

[14] P. Tsarouhas, T. Varzakas \& I. Arvanitoyannis, "Reliability and maintainability analysis of strudel production line with experimental data: a case study" J. Food Eng. 91 (2009) 250.

[15] F.J.G. Carazas \& G.F.M. Souza, "Availability analysis of gas turbines used in power plants", Int. J. of Thermodynamics 12 (2009) 28.
[16] A. K. Lado \& V. V. Singh, "Cost assessment of complex repairable system consisting two subsystems in series configuration using Gumbel Hougaard family copula”, International Journal of Quality \& Reliability Management 36 (2019) 1683. https://doi.org/10.1108/IJQRM-12-20180322 .

[17] V. V. Singh, P. K. Poonia \& A. H. Adbullahi, "Performance analysis of a complex repairable system with two subsystems in series configuration with an imperfect switch", J. Math. Comput. Sci., 10 (2020) 359. DOI: $10.28919 / \mathrm{jmcs} / 4399$

[18] I. Yusuf, A. L. Ismail \& V. V. Singh et al., "Performance Analysis of Multi-computer System Consisting of Three Subsystems in Series Configuration Using Copula Repair Policy”, SN Comp. Sci. 1 (2020) 241. https://doi.org/10.1007/s42979-020-00258-0.

[19] D. Raghav, D. Rawal, I. Yusuf, R. H. Kankarofi \& V. Singh, "Reliability Prediction of Distributed System with Homogeneity in Software and Server using Joint Probability Distribution via Copula Approach", Reliability: Theory \& Applications, 16 (2021) 217.https://doi.org/10.24412/1932-2321-2021-161-217-230 